\title{
Comportamiento dinámico del biogas en el relleno sanitario del cantón Gonzalo Pizarro, Ecuador
}

\author{
Juan Pablo Morales Corozo ${ }^{1}$ \\ j.p.shevarojo@gmail.com \\ ORCID: https://orcid.org/0000-0002-4538-4488
}

\section{RESUMEN}

Dentro de las competencias exclusivas del Gobierno Autónomo Descentralizado Municipal del cantón Gonzalo Pizarro se encuentra la gestión integral de los desechos sólidos dentro de lo cual se realizan las actividades de recolección, transporte, tratamiento y disposición final de los desechos sólidos. El objetivo de este trabajo de investigación es calcular la cantidad de biogas generado en el relleno sanitario y determinar el nivel expansivo de los olores del mismo en el área de influencia directa para estimar los daños ambientales ocasionados. Para analizar el comportamiento dinámico de los biogases existen una diversidad de métodos los cuales varían al tomar en cuenta las variables que se incluye dentro de los modelos matemáticos desarrollándose para cada caso determinado. "Es posible que un modelo únicamente sea óptimo para un determinado tipo de vertedero y para unas condiciones climáticas particulares" (Lecuona, 2019, p. 13). El modelo gaussiano es muy cambiante y con los parámetros básicos nos muestra predicciones muy acertadas ajustadas a la realidad del sitio en estudio, a la vez la emisión del biogas es baja motivo por el cual dificulta el aprovechamiento energético del mismo motivo por el cual las chimeneas de desfogue es una de la alternativa viable aplicada a un relleno sanitario pequeño con capacidad de 4 toneladas al día de desechos sólidos. La debilidad del modelo de dispersión de olores es que no cuentan con variaciones de parámetros meteorológicos, siendo la incertidumbre de aplicabilidad considerable.

Palabras clave: desechos sólidos; biogases; modelos matemáticos de dispersión de olores

\footnotetext{
${ }^{1}$ Candidato a Doctor del programa Doctorado en Ambiente y Desarrollo, de la Universidad Nacional Experimental de los Llanos Occidentales "Ezequiel Zamora", Director de Gestión de Ambiente del Gobierno Autónomo Descentralizado Municipal del cantón Gonzalo Pizarro, de la provincia de Sucumbíos Ecuador. Ingeniero Químico. Magíster de alta dirección y varios posgrados
} 


\title{
Dynamic behavior of biogas in the canton Gonzalo Pizarro sanitary landfill, Ecuador
}

\begin{abstract}
Within the exclusive competences of the Municipal Autonomous Decentralized Government of the Gonzalo Pizarro canton is the integral management of solid waste within which the activities of collection, transport, treatment and final disposal of solid waste are carried out. The objective of this research work is to calculate the amount of biogas generated in the sanitary landfill and determine the expansive level of the odors of the same in the area of direct influence to estimate the environmental damage caused. To analyze the dynamic behavior of biogases there are a variety of methods which vary by taking into account the variables that are included in the mathematical models being developed for each specific case. "It is possible that a model is only optimal for a certain type of landfill and for particular climatic conditions" (Lecuona, 2019, p. 13). The Gaussian model is very changeable and with the basic parameters it shows us very accurate predictions adjusted to the reality of the site under study, at the same time the emission of biogas is low, which makes it difficult to use energy for the same reason that the chimneys. dump is one of the viable alternative applied to a small sanitary landfill with a capacity of 4 tons per day of solid waste. The weakness of the odor dispersion model is that it does not have variations in meteorological parameters, with considerable applicability uncertainty.
\end{abstract}

Keywords: solid waste; biogas; mathematical models of odor dispersion

Artículo recibido: 05 octubre. 2021 Aceptado para publicación: 02 noviembre 2021 Correspondencia: j.p.shevarojo@gmail.com Conflictos de Interés: Ninguna que declarar 


\section{INTRODUCCIÓN}

En el mundo, así como en el Ecuador, manejar los desechos sólidos, es un reto y a la vez "un problema por la inadecuada recolección, clasificación y disposición final de los mismos, provocando problemas ambientales y sociales" (Chela, 2016, p. 12). El manejo adecuado de los desechos sólidos no peligrosos se encuentran descritos en el Código Orgánico Ambiental, y atribuye la competencia exclusiva para la gestión a los gobiernos autónomos municipales. En el año 2014, luego de la aparición del Programa Nacional de la Gestión Integral de los Desechos Sólidos, activó varias respuestas en el mejoramiento del manejo a nivel nacional donde los gobiernos municipales elaboraron ordenanzas para la recolección, transporte, almacenamiento, tratamiento y disposición final. Calero, et. al., (2021) sostiene que los desechos sólidos generan el 3,95\% de las emisiones de los gases de efecto invernadero. "En Ecuador, en el 2017 se estimó una generación de 0,78 kg/hab./día y de 0,86 kg/hab./día para el año 2018" (Kasa, 2018, p. 50).

La planificación para una adecuada gestión integral de los desechos sólidos, involucra una serie de actividades tendientes a conocer la situación actual, así como definir los criterios básicos para su proyección, sustentabilidad y sostenibilidad a corto, mediano y largo plazo, siendo cada una de estas concatenadas y de igual importancia. Los desechos sólidos no tienen las mismas características, el volumen o tipo varian dependiendo algunos factores como son: el ámbito territorial, el tipo de generador y las condiciones climáticas.|

El cantón Gonzalo Pizarro, se encuentra ubicado en la provincia de Sucumbíos, tiene una extensión de 224.208,94 hectáreas, y se encuentra limitada al norte con el cantón Sucumbíos, al sur el cantón Chaco, al este el cantón Cascales y al oeste los cantones de Pimampiro y refleja un estado de conservación del paisaje que se ve modificado por la actividad antrópicas que según Alvear, (2021) el 76,96\% del territorio cantonal se encuentra dentro del Sistema Nacional de Áreas Protegidas, motivo por el cual se debe tomar en cuenta medidas para evitar la contaminación de los recursos naturales a traves del manejo adecuado de los desechos sólidos.

Dentro de las competencias exclusivas del Gobierno Autónomo Descentralizado Municipal del cantón Gonzalo Pizarro se encuentra la gestión integral de los desechos sólidos dentro de lo cual se realizan las actividades de recolección, transporte, tratamiento y disposición final de los desechos sólidos. Diariamente ingresan al relleno sanitario 
aproximadamente 5 toneladas, de las cuales un $10 \%$ son aprovechables y el resto ingresa a una celda de disposición final. Si bien los desechos sólidos se les da un manejo adecuado pero se desconoce el nivel de afectación de los biogases a los moradores del sector, a la vez no se ha realizado una evaluación de los daños ambientales debido a esta emisión. El objetivo de este trabajo de investigación es calcular la cantidad de biogas generado en el relleno sanitario y determinar el nivel expansivo de los olores del mismo en el área de influencia directa para estimar los daños ambientales ocasionados.

El crecimiento gradual de los desechos sólidos son el resultado de una variación de la dinámica urbana donde se expande los sectores debido a crecimiento poblacional, existiendo una relación "directa entre la generación y caracterización de residuos con la cantidad de habitantes" (Madrigal, Quishpe, y Vargas, 2018, p. 44). El manejo de los desechos sólidos de la mayoría de los gobiernos municipales es a través de vertederos o tambien llamados rellenos sanitarios donde se depositan para ser tratados, estos a la vez generan cantidades de biogases que comprende una mezcla de metano, disoxido de carbono, compuestos volátiles y una diminuta cantidad de monoxido de carbono y óxido nitroso. (Madrigal, Quishpe, y Vargas, 2018) dentro de su investigación sostienen que el metano "contrubuye aproximadamente un 3 a un 4 por ciento de las emisiones de gases de efecto invernadero antropogénicas mundiales anuales" (p. 45).

(Kiss y Aguilar, 2006) manifiestan que la calidad del biogas producto de la descomposición de los desechos sólidos depende de las condiciones meteorológicas y climáticas como la precipitación, vientos y temperatura; las propiedades físicoquímicas que "varían según el sitio, y tambien en el tiempo, resultando, por ende lixiviado y biogas" (p. 40), siendo la cantidad de contaminantes función de la composición química de los desechos sólidos; la tecnología utilizada para el manejo, tratamiento y disposición final influyen en la velocidad de descomposición; por ultimo la edad del relleno es uno de los factores importantes que "depende no solo de las características de los residuos dispuestos y de las condiciones ambientales y tecnológicas, sino tambien de la capacidad de reacción de los materiales depositados, la cual a largo plazo va disminuyendo" (Kiss y Aguilar, 2006, p. 41). La descomposición de los deschos sólidos se divide en las siguientes fases:

La fase I anaeróbica se da al momento de la conformación de la celda diaria donde el aire atrapado se consume de forma rápida, donde debido a las reacciones exotérmicas que 
provocan subidas de temperatura generan dioxido de carbono, hasta el agotamiento del oxígeno.

La fase II transición, donde una vez consumido el oxígeno se da un proceso de fermentación, generandose lixiviados que continen ácidos orgánicos y el biogas se encuenra conformado por metano y dioxido de carbono. "Mientras el vertedero se convierte en anaerobio, el nitrato y el sulfato, que pueden servir como receptores de electrones en reacciones de conversión biológica, a menudo se reducen a gas nitrógeno y sulfuro de hidrógeno" (Lecuona, 2019, p. 10).

La fase III ácida, aquí "predominan las condiciones totalmente anaerobias" (Kiss y Aguilar, 2006, p. 44). En esta fase se da la mayor producción de metano y se estabiliza la concentración de biogas, debido a que los ácidos orgánicos se transforman en volátiles y disminuye en mayor proporción el dioxido de carbono.

La fase IV fermentación del metano, los microorganismos se encargan de la conversión de gases de hidrógeno y ácido acético en biogas que se tornan estables, se eleva el pH del lixiviado y disminuye la concentración de DQO y $\mathrm{DBO}_{5}$.

La fase $\mathbf{V}$ maduración, donde la producción de metano disminuye "el bióxido de carbono se sustituye por nitrógeno y oxígeno conforme se introduce el aire atmosférico a través de las capas superficiales del relleno" (Kiss \& Aguilar, 2006, p. 44). En esta fase disminuye la humedad, “los nutrientes diluidos en el lixiviado y la lenta degradación de los sustratos disponibles en el medio sólidos provoca una significativa disminución en la generación de gas" (Lecuona, 2019, p. 12).

\section{Gráfico 1 Fases de descomposición de los desechos sólidos y generación de biogas}

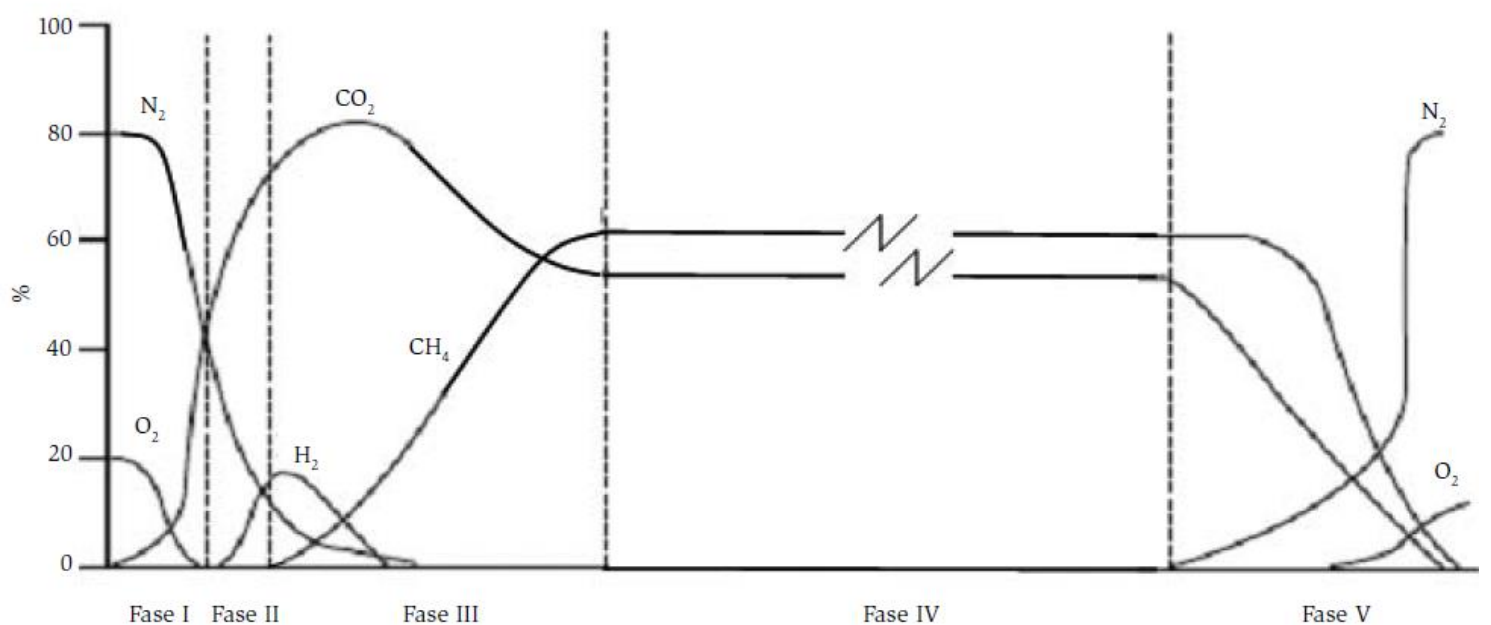

Fuente: (Kiss y Aguilar, 2006) 
La investigación sobre el biogas en el relleno sanitario es una preocupación constante dentro del manejo de los desechos sólidos. Debido a las reacciones bioquímicas el proceso con el cual se originan los biogases es muy complejo donde uno de los principales actores son los microorganismos metanogenénicos, los cuales son muy sensibles al variaciones ambientales. La mayor parte de los investigadores evaluan la fase de metanogénesis a partir de la tasa de producción de metano. "El cálculo de la tasa de generación de biogas en un relleno sanitario es de especial interés para poder justificar la compra de materiales e insumos para el manejo del gas" (Ávila, 2021, p. 15). Es importante conocer la cantidad de biogas producido dentro del relleno sanitario para tomar medidas para su manejo adecuado y en el caso que aplique realizar un aprovechamiento energético de los mismos evitando su emisión a la atmósfera.

\section{MATERIALES Y MÉTODOS}

La información de los desechos sólidos recolectados en las diferentes parroquias del cantón Gonzalo Pizarro, que ingresan al relleno sanitario. En este estudio se llevó a cabo inicialmente una revisión sistemática de la literatura presente a partir de la gestión integral de los desechos sólidos y los estudios de la generación de los biogases. El sitio donde se realizó la investigación fue en el relleno sanitario del cantón Gonzalo Pizarro, ubicado en el recinto Nuevo Paraíso, en la parroquia Lumbaqui, con una extensión de 11,2 hectáreas, cuanta con una Licencia Ambiental, emitida por el Ministerio del Ambiente mediante Resolución No. 361 del 01 de septiembre de 2010, hasta la fecha se han implantado 5 celdas de disposición final, con respecto a las condiciones climáticas se tiene una temperatura promedio de $23^{\circ} \mathrm{C}$, una precipitación anual promedio de $5000 \mathrm{~mm}$, y una humedad relativa de $80 \%$. El relleno sanitario recibe diariamente un promedio de 4 toneladas provenientes de las parroquias Lumbaqui, Gonzalo Pizarro, El Reventador y Puerto Libre, la producción per cápita es de 0,337 Kg/hab/día.

Para analizar el comportamiento dinámico de los biogases existen una diversidad de métodos los cuales varían al tomar en cuenta las variables que se incluye dentro de los modelos matemáticos desarrollándose para cada caso determinado. "Es posible que un modelo únicamente sea óptimo para un determinado tipo de vertedero y para unas condiciones climáticas particulares" (Lecuona, 2019, p. 13). El modelo aplicado para nuestra investigación es el propuesto por Roben, (2002) utilizado en el Gobierno Municipal de Loja el cual toma en cuenta la generación de metano en un principio por 
tratarse de un vertedero pequeño es baja y su producción aumenta gradualmente hasta disminuir.

$$
G_{\text {max }}=1,868 * C_{\text {org }} *(0,014 T+0,28) *\left(1-10^{-k t}\right)
$$

\section{Donde}

$C_{\text {org }}$ Cantidad de carbono orgánico de los desechos sólidos

T Temperatura $\left({ }^{\circ} \mathrm{C}\right)$

$k$ Constante de biodegradación

Los modelos de dispersión atmosférica de los biogases y su variación con el tiempo, se lo puede obtener a partir de una ecuación de continuidad, el cual "expresa el balance entre las variaciones de la concentración de una traza y el efecto de flujos de transporte, fuentes y sumideros" (Cabrera, 2012, p. 12). Estos modelo de dispersión utilizan varios métodos con la finalidad de predecir la carga contaminante en el punto más alejado de la fuente emisora, Cabrera, (2012) manifiesta que en los modelos matemáticos se incluye modelos gausianos para la continuidad, ya que son comunes su aplicación para "problemas de dispersión contaminantes no reactivos de fuentes puntuales" (p. 14). Existen modelos de dispersión de emisiones olfativas la cual depende de concentraciones de gases olfatorios, estas se pueden determinar por medio del siguiente modelo matemático:

$$
C_{(x y z)} \frac{10^{6}}{3600 * 2 * p} * \frac{Q}{u_{h} * \delta_{y} \delta_{z}} * e^{\left(\frac{y^{2}}{2 \delta_{y}^{2}}\right)} * e^{\left(\frac{-(z-h)^{2}}{2 \delta_{z}^{2}}\right)}+e^{\left(\frac{-(z+h)^{2}}{2 \delta_{z}^{2}}\right)}
$$

\section{Donde}

$Q \quad$ Tasa de emisión de biogas

$\delta_{y} ; \delta_{z} \quad$ Parámetros de dispersión

$u_{h} \quad$ Rapidez promedio de la altura de emisión

$p \quad$ Peralte del caudal del gas 


\section{RESULTADOS}

Tabla 1 Biogas y la dispersión de emisiones olfativas

\begin{tabular}{|c|c|c|c|c|c|}
\hline Fecha & Mes & $\begin{array}{c}\text { Cantidad de } \\
\text { desechos } \\
(\mathrm{kg})\end{array}$ & $\begin{array}{l}\text { Cantidad } \\
\text { de biogas } \\
\mathbf{G}_{\text {real }}(\mathbf{k g})\end{array}$ & $\begin{array}{c}\text { Volumen de } \\
\text { biogas } \\
\text { greal }_{(\mathrm{m} 3)}\end{array}$ & $\begin{array}{c}\text { Dispersión de } \\
\text { emisiones } \\
\text { olfativas } \\
\text { c } \mathrm{xyz}(\mathrm{uo} / \mathrm{m} 3)\end{array}$ \\
\hline $1 / 6 / 2021$ & JUNIO & 3870 & 97,96 & 78,368 & 0,0000 \\
\hline $2 / 6 / 2021$ & JUNIO & 2570 & 65,05 & 52,04 & 0,4704 \\
\hline $3 / 6 / 2021$ & JUNIO & 5400 & 261,34 & 209,072 & 0,0000 \\
\hline $4 / 6 / 2021$ & JUNIO & 4860 & 337,53 & 270,024 & 0,4704 \\
\hline $5 / 6 / 2021$ & JUNIO & 4370 & 387,41 & 309,928 & 0,0001 \\
\hline $7 / 6 / 2021$ & JUNIO & 3760 & 399,17 & 319,336 & 0,0000 \\
\hline $8 / 6 / 2021$ & JUNIO & 3720 & 454,34 & 363,472 & 0,1833 \\
\hline $9 / 6 / 2021$ & JUNIO & 3040 & 415,57 & 332,456 & 0,0000 \\
\hline $10 / 6 / 2021$ & JUNIO & 6610 & 991,39 & 793,112 & 0,8282 \\
\hline $11 / 6 / 2021$ & JUNIO & 4310 & 698,65 & 558,92 & 0,0090 \\
\hline $12 / 6 / 2021$ & JUNIO & 2340 & 405,17 & 324,136 & 0,0000 \\
\hline $14 / 6 / 2021$ & JUNIO & 6610 & 1211,11 & 968,888 & 0,0011 \\
\hline $15 / 6 / 2021$ & JUNIO & 6210 & 1194,9 & 955,92 & 0,8282 \\
\hline $16 / 6 / 2021$ & JUNIO & 920 & 184,73 & 147,784 & 0,0011 \\
\hline $17 / 6 / 2021$ & JUNIO & 5580 & 1163,1 & 930,48 & 0,0011 \\
\hline $18 / 6 / 2021$ & JUNIO & 8030 & 1729,76 & 1383,808 & 0,0000 \\
\hline $19 / 6 / 2021$ & JUNIO & 4230 & 938,09 & 750,472 & 0,0011 \\
\hline $21 / 6 / 2021$ & JUNIO & 7890 & 1795,52 & 1436,416 & 0,0090 \\
\hline $22 / 6 / 2021$ & JUNIO & 6070 & 1413,44 & 1130,752 & 0,8282 \\
\hline $23 / 6 / 2021$ & JUNIO & 1310 & 311,36 & 249,088 & 0,0000 \\
\hline $24 / 6 / 2021$ & JUNIO & 4450 & 1077,25 & 861,8 & 0,1833 \\
\hline $25 / 6 / 2021$ & JUNIO & 3890 & 957,29 & 765,832 & 0,1833 \\
\hline $26 / 6 / 2021$ & JUNIO & 4270 & 1066,43 & 853,144 & 0,0090 \\
\hline $28 / 6 / 2021$ & JUNIO & 7330 & 1855,12 & 1484,096 & 0,0490 \\
\hline $29 / 6 / 2021$ & JUNIO & 4460 & 1142,34 & 913,872 & 0,0000 \\
\hline $30 / 6 / 2021$ & JUNIO & 1300 & 336,58 & 269,264 & 0,0011 \\
\hline
\end{tabular}

Fuente: Morales, (2021)

De la investigación realizada el mes de junio de 2021, observa en la tabla 1 que el promedio de desechos sólidos que ingresan al relleno sanitario es de 4.515,38 $\mathrm{Kg}$, el día donde que realiza el recorrido de la ruta de las comunidades es donde ingresan menor cantidad de desechos sólidos al vertedero teniendo el menor valor de 920,00 Kg, y los días donde ingresa mayor cantidad es cuando se realiza el recorrido de dos rutas y se suma 
ingresados por las empresas directamente siendo el valor más alto 8.030,00 Kg. El modelo de dispersión de emisiones olfativas al calcularlo nos muestra valores inferiores a 0,70 $\mathrm{UO} / \mathrm{m}^{3}$, mostrándonos que la afectación al área de influencia directa no es significativa, a la vez el manejo de los desechos en la celda de disposición final es adecuado.

\section{CONCLUSIONES}

Los moradores del sector del área de influencia directa no se han visto afectados por los olores emitidos por los desechos sólidos, sus valores diarios son menores a $0.7 \mathrm{UO} / \mathrm{m} 3$, siendo los resultados obtenidos del modelo matemático de dispersión atmosférica coherentes con la realidad, a la vez nos muestra el manejo adecuado realizado en el vertedero.

La cantidad de biogas promedio por día es de $642,79 \mathrm{~m}^{3}$, el cual no tiene un efecto considerable en el medio ambiente, esto podría deberse a las condiciones del sitio donde se encuentra asentado el vertedero donde se tiene una humedad relativa de $80 \%$, temperaturas promedio de $28^{\circ} \mathrm{C}$, y precipitaciones anuales superiores a $5000 \mathrm{~mm}$, lo que influiría en la deficiencia en la emisión. Otro aspecto que se ha observado en el momento de realizar la investigación es que no existe variaciones en los cultivos que se desarrollan en el área de influencia directa que puedan deberse a reacciones producto de las emisiones que influyan en la degradación del suelo y disminuyan la calidad de los cultivos.

El modelo gaussiano es muy cambiante y con los parámetros básicos nos muestra predicciones muy acertadas ajustadas a la realidad del sitio en estudio, a la vez la emisión del biogas es baja motivo por el cual dificulta el aprovechamiento energético del mismo motivo por el cual las chimeneas de desfogue es una de la alternativa viable aplicada a un relleno sanitario pequeño con capacidad de 4 toneladas al día de desechos sólidos. La debilidad del modelo de dispersión de olores es que no cuentan con variaciones de parámetros meteorológicos, siendo la incertidumbre de aplicabilidad considerable.

\section{REFERENCIAS BIBLIOGRAFICAS}

Alvear, C. (2021). Plan de Desarrollo y Ordenamiento Territorial. Edxitoriales Quevedo. Andrade, A., Restrepo, A., \& Tibaquirá, J. (2017). Estimación de biogas de relleno sanitario, caso de estudio: Colombia. Entre Ciencia e Ingeniería, año 12 (vol 12), no. 23, 40 - 47. doi:https://doi.org/10.31908/19098367.3701 
Ávila, M. (2021). Determinación de la cantidad y composición del biogas producido en un relleno sanitario a escala de laboratorio en la Universidad Libre Sede Bosque Popular. Universidad Libre sede Bosque Popular.

Cabrera, J. (2012). Aplicación de un modelo de dispersión atmosférica. Pontificia Universidad Católica de Valparaíso.

Calero, et. al. (2021). Gestión local de cambio climático: planificación participativa y gobernanza territorial. FLACSO.

Cárdenas, et. al. (2013). Cálculo del biogas generado en los sitios de disposición final de los residuos sólidos urbanos en el Estado de México. VSUR - DEDISA, 1 - 6.

Chela, J. (2016). Manejo integral de los desechos sólidos en la Universidad Estatal de Bolivar, cantón Guaranda, provincia Bolivar, periodo mayo - septiembre. Universidad Estatal de Bolívar.

Colomer, et. al. (2016). Emisiones gaseosas de un relleno sanitario en México, comparación con los modelor de generación de biogas. Rev. Int. Contam. Ambie. 32 (Especial Residuos Sólidos), 113 - 123. doi:10.20937/RICA.2016.32.05.08

Córdova, V., Blanco, G., \& Santalla, E. (2009). Modelación de la generación de biogas en rellenos sanitarios. Avances en Energías Renovables y Medio Ambiente vol. 13, $69-77$.

Escamilla, P. (2019). Eficiencia y confiabilidad de modelos de estimación de biogas en rellenosa sanitarios. LA GRANJA: Revista de Ciencias de la Vida 29(1), 32 - 44. doi:doi.org/10.17163/lgr.n29.2019.03

González, Y., Gato, T., Guillot, R., \& Pires, L. (2015). Determinación del potemcial energético de los residuos sólidos urbanos en tres municipios de la provincia de Luanda, Angola. Tecnología Química, vol. XXXV, núm. 1,, 35 - 44.

Kasa, S. (2018). What a waste 2.0: A Global Snapshot of solid waste management to 2050. World Bank Group.

Kiss, G., \& Aguilar, E. (2006). Los productos y los impactos de la descomposición de residuos sólidos urbanos en los sitios de disposición final. Gaceta ecológica 79, $39-51$.

Lecuona, A. (2019). Modelización matemática de la producción de biogas en vertederos de residuos sólidos urbanos. Universidad Politécnica de Valencia. 
Madrigal, G., Quishpe, J., \& Vargas, Y. (2018). Cálculo de la generación de biogas para el relleno sanitario de la ciudad de Juliaca, utilizando el modelo LandGEM Versión 3.02 de la USEPA y estimación del potencial de producción eléctrica. Revista de Investigación: Ciencia, Tecnología y Desarrollo (2018) Volumen 4 Número (2):, 42 - 55. doi:https://doi.org/10.17162/rictd.v4i2.1096

Panesco, A. (2011). Análisis del biogas captado en un relleno sanitario como combustible primario para la generación de energía electrica. Scientia et Technica Año XVII, No 47, 23 - 30.

Pico, L. (2018). Simulación del proceso de digestión anaeróbia para predecir la producción de. Universidad Estatal Amazónica.

Vera, et. al. (2015). Potencial de generación de biogas y energía eléctrica. Parte II: residuos sólidos urbanos. Ingeniería Investigación y Tecnología, volumen XVI (número 3),, 471 - 478. 